\title{
Medical decision making about long-term artificial nutrition after severe stroke: a case report
}

\author{
Amber R. Comer ${ }^{1}$, Linda S. Williams ${ }^{2}$, Stephanie L. Bartlett ${ }^{1}$, Lynn E. D'Cruz ${ }^{1}$, Alexia M. Torke ${ }^{3}$ \\ ${ }^{1}$ Department of Health Sciences, Indiana University School of Health and Human Sciences, Indianapolis, IN, USA; ${ }^{2}$ Department of Neurology, \\ Indiana University School of Medicine, Indianapolis, IN, USA; ${ }^{3}$ Department of Internal Medicine and Geriatrics, Indiana University School of \\ Medicine, Indianapolis, IN, USA \\ Correspondence to: Amber R. Comer, PhD, JD. Department of Health Sciences, Indiana University School of Health and Human Sciences, 1050 \\ Wishard Blvd., RG 3034, Indianapolis, IN 46202, USA. Email: comer@iu.edu.
}

\begin{abstract}
Choosing to use a percutaneous endoscopic gastrostomy (PEG tube) for long term artificial nutrition in the setting of inadequate oral intake after stroke is complex because the decision must be made in a relatively short amount of time and prognosis is often uncertain. This case study utilized interviews with attending and resident neurologists, and surrogate medical decision makers in order to examine how neurologists and surrogate medical decision makers approached the decision to either receive a PEG tube or pursue comfort measures after severe stroke in two patients. Although these two patients presented with similar clinical characteristics and faced similar medical decisions, different decisions regarding PEG tube placement were made. Major challenges included physicians who did not agree on prognosis and surrogates who did not agree on whether to place a PEG tube. These cases demonstrate the importance of the role of the surrogate medical decision maker and the necessity of physicians and surrogate medical decision makers approaching the complex decision of PEG tube placement after stroke together. Additionally, these cases highlight the differing views on what defines a good quality of life and show the vital importance of highquality goals of care conversations about prognosis and quality of life when deciding whether to place a PEG tube after severe stroke.
\end{abstract}

Keywords: Medical decision making; goals of care conversations; quality of life; case report

Submitted Oct 23, 2020. Accepted for publication Mar 30, 2021.

doi: 10.21037/apm-20-2094

View this article at: http://dx.doi.org/10.21037/apm-20-2094

\section{Introduction}

Medical decision making after severe stroke is complicated as it often involves life and death decisions that must be made in a relatively short amount of time despite an uncertain prognosis. Preference-sensitive decisions made by surrogate medical decision makers can result in stroke patients experiencing prolonged suffering and aggressive treatments they would not have chosen for themselves (1-5). One important decision encountered in patients with severe stroke with affected swallowing function is whether to use a percutaneous endoscopic gastrostomy (PEG) tube to supply artificial nutrition and hydration.

Choosing to use a PEG tube for long term artificial nutrition in the setting of inadequate oral intake after stroke is a complex decision (6-8). There are differing perspectives on quality of life after the placement of a PEG tube. For instance, some patients and surrogates believe that death would be preferable to having a PEG tube, especially if the patient is expected to have very poor quality of life (9-19). On the other hand, some patients and surrogates believe that living is preferable to death, regardless of the patient's quality of life, and that receipt of artificial nutrition does not significantly worsen overall quality of life (9-19). Although it is ethically acceptable to remove a PEG tube after placement, this decision is often challenging as PEG tubes may be regarded as ordinary care (care that must be provided to the patient) by many groups, even when the patient is experiencing poor quality of life (17). The purpose 
of this case study was to examine how neurologists and surrogate medical decision makers approached the decision for a patient to receive artificial nutrition via a PEG tube after severe stroke.

We present the study in accordance with the CARE reporting checklist. Available at http://dx.doi.org/10.21037/ apm-20-2094.

\section{Case presentation}

Two incapacitated patients (Patient A and Patient B) with similar National Institutes of Health Stroke Scale scores (NIHSS), stroke type and severity, and prior level of function, who were facing a medical decision regarding the placement of a PEG tube were identified. We conducted an in-depth comparison of these two patients because of the ethical and communication issues raised by the decisionmaking process in each case. The patients' surrogate medical decision makers were consented for enrollment in this study. Physicians were also consented for this study. The University IRB approved this study. Both patient's surrogates were consented for participation in this study.

Chart review of the patient's medical records, semistructured interviews with the patient's attending and resident neurologists, as well as with the patient's surrogate medical decision makers were conducted. Interviews were conducted during the first week of the patient's hospitalization. The interviewer asked the patient's physician and surrogates to identify what they believed the best case, worst case, and most likely outcome was for the patient. Additionally, the interviews allowed the physicians and surrogates to discuss barriers to medical decisions. All interviews were audio recorded and transcribed. Members of the study team identified issues with medical decision-making regarding PEG tube placement as they emerged from both the coded transcripts and chart review information.

Interviews were obtained from Patient A's daughter, two attending neurologists, and one resident neurologist. Interviews were obtained from Patient B's sister and son, as well as one attending and one resident neurologist. Patient A included two attending neurologists as their major medical decision occurred at a time when the first attending was transitioning off of service.

\section{Patient A}

\section{Clinical and social characteristics}

Patient A was an 81-year-old retired woman with a history of atrial fibrillation, osteoarthritis, $\mathrm{CAD}$, and anxiety who presented with a left middle cerebral artery (MCA) stroke and an initial NIHSS severity score of 10 (indicating moderate to severe impairments). Patient A received both tissue plasminogen activator (tPA) and thrombectomy. She was subsequently admitted to the ICU and placed on mechanical ventilation. The patient decompensated during hospitalization and palliative care was consulted on day 6 of hospitalization in order to discuss goals of care, code status, and comfort measures. The patient was extubated and made DNR on day 8 of hospitalization. After extubation, Patient A faced a medical decision regarding the placement of a PEG tube due to persistent inability to swallow. The patient was unable to communicate and lacked decision making capacity. Patient A's son was initially making medical decisions for this patient and requested aggressive interventions; however, he often did not keep scheduled meetings with the clinical team. Additionally, clinical care notes documented the care team's suspicion that the son used illegal drugs and was using the patient's social security income to purchase drugs. On day 6 of hospitalization, Patient A's daughter was located and contacted by the palliative care team and informed of the pending medical decision of PEG tube placement. Patient A's daughter, who lived out of town, came to the hospital and assumed medical decision-making responsibilities. At that point, the patient's son stopped participating in the patient's medical decisions. The daughter was legally able to serve as the patient's surrogate under the health care consent law of the state in which the patient was hospitalized, which allows physicians to remove surrogates who are not acting or making medical decisions in the patient's best interest.

\section{Physician prognosis}

Both the attending and resident physicians involved in this patient's case indicated that the best outcome for this patient would not include a return to baseline; however, the range of impairment the patient would likely endure varied from being able to communicate and engage in activities of daily living to never being able to walk or talk. Attending 1 indicated that the worst outcome for this patient would be if she were to be kept alive artificially with a tracheostomy and a PEG tube without being able to enjoy life. "That's a bit of a value judgment. I think the worst possible outcome would be her being kept alive artificially on a tracheostomy and a PEG tube, but not having any ability to enjoy life."-Attending 1 . Both Attending 2 and the medical resident indicated that the worst outcome would be further strokes and death. The neurology notes 
from this patient state that the most likely outcome was "that the patient would likely need long term institutional care given her severe cognitive deficits." The neurology team recommended long term placement in a nursing home.

\section{Surrogate prognosis}

Patient A's daughter indicated that she believed the worst outcome for her mother would be that she "goes to a nursing bome and lays in a bed for montbs". Patient A's daughter indicated that she preferred her mother's death to watching her mother live a life with no quality. The surrogate indicated that although she wished to pursue comfort measures only, she was not confident in her choice due to conflicting information given to her by the palliative care and neurology teams. The patient stated that the neurology team expressed optimism and "made [her] feel like [she] should wait" to make a decision on pursuing comfort measures. Whereas the palliative care team recommended a transition to comfort measures.

\section{Goals of care}

The daughter stated the palliative care team had indicated that comfort measures was the appropriate plan; however, several hours later, neurology questioned the daughter's choice to pursue comfort measures as the neurology team had a more optimistic prognosis. "I got mixed reviews from two different doctors... I'm confused... I felt resolution with (comfort measures) and then when I spoke to the neurologist, I felt judged that she was implying like you're going to give up."Patient A's daughter.

\section{Patient outcome}

The daughter decided against placement of a PEG tube. The patient was placed on inpatient hospice and died on day 16 of hospitalization.

\section{Patient B}

\section{Clinical characteristics}

Patient B is a 58 -year-old man with a history of prior right cerebrovascular event with residual spastic paralysis, and hypertension who presented with a left MCA occlusion and an initial NIHSS severity score of 10. Patient B was ineligible to receive tPA due to unknown time of last normal and the patient did not receive thrombectomy. Patient B was subsequently admitted to the ICU with a full code status. Patient B faced a medical decision regarding the placement of a PEG tube due to dysphagia. The patient's medical chart had a note from his neurologist during the initial hospitalization which stated that when the patient was asked whether he wanted to receive a feeding tube, the patient shook his head no. Although Patient B was able to nod his head when asked yes or no questions, he was determined to lack medical decision-making capacity because of his aphasia and severe neurologic injury. The patient's son initially served as the patient's medical decision maker; however, his son felt unsure of his ability to make medical decisions and deferred decision making to his aunt, the patient's sister. Palliative care was consulted on day 3 of hospitalization in order to discuss goals of care. The patient was made a DNR on day 27 of the hospitalization.

\section{Physician prognosis}

Both the attending and resident neurologists on this case indicated that the best case scenario for this patient would be if he were able to return home with the assistance of a home health aide or skilled nursing visits. The worst case scenario was recurrent stroke and death. The neurologist believed the patient would likely need placement in a longterm care facility.

\section{Surrogate prognosis}

Patient B's son (surrogate 1), indicated that he did not believe that his father would ever return to baseline or regain his mobility, even in the best case scenario. Furthermore, Patient B's son indicated that death was the worst case scenario but that he did not believe that his father would have wanted any surgeries or procedures like a PEG tube during this hospitalization.

Patient B's sister (surrogate 2), believed that in the best case scenario the patient would regain some speech and ability to walk and swallow. Surrogate 2 indicated the worst case scenario would be for the patient to be in a vegetative state and lack quality of life. As an additional concern, Patient B's sister stated that she believed the hospital would try to give up on her brother because he was poor and Black. Additionally, she indicated that her brother was a fighter and they should not give up because he had recovered (although not back to baseline) from a prior stroke.

\section{Patient outcome}

Patient $B$ received a PEG tube on day 8 of hospitalization and was discharged to inpatient rehabilitation facility on day 16; however, he was subsequently readmitted to the hospital the next day for chest pain with tachycardia which was later diagnosed as sympathetic storms. During the readmission, 
the patient pulled out his PEG tube and the PEG tube was replaced the next day. At the time that the patient pulled out the PEG tube, an internal medicine doctor noted in the patient's medical record that "the patient still seemed to shake bis head 'no' to the idea of a feeding tube, but apparently a family member was able to get him to agree. I'm unsure who has given (or will give) consent on behalf of the patient. A trial of PEG tube feedings for support is reasonable but I'm afraid that the patient's quality of life will not improve much and it is often difficult to decide to stop using the PEG for feeding once it is in. If he (and the family) eventually decide to stop using the PEG tube feeds, it can be used for comfort meds at the end of life." Throughout the patient's hospital stay, palliative care notes indicate that they repeatedly discussed stopping the patient's tube feeds, additionally the PEG tube was either pulled out by the patient or fell out and was replaced four times. Nevertheless, the family did not want to stop feeds.

In total, Patient B spent an additional 148 days in the hospital prior to being discharged to a subacute nursing facility. At the time of discharge, the patient was noted to be unresponsive and had not regained the ability to speak, walk, or swallow.

All procedures performed in studies involving human participants were in accordance with the ethical standards of the institutional and/or national research committee(s) and with the Helsinki Declaration (as revised in 2013). Written informed consent was obtained from the patient or their surrogate.

\section{Discussion}

Although these two patients presented with similar clinical characteristics and faced similar medical decisions, these patients had very different outcomes. Even though both patients had surrogates who indicated that they did not want to see the patient have a poor quality of life, different decisions were made regarding PEG tube placement. These cases highlight the complexity of decisions to pursue PEG tube after stroke and differing views on what defines a good quality of life. Each case had its own major challenges during the decision-making process. In case A, the surrogate felt like she was pitted between the palliative care physician and the neurologist, both of whom had differing opinions on whether the patient should have a PEG tube placed. The major challenge in Patient B's care was that the patient and his son both expressed reservations about having a PEG tube placed; however, the patient's sister assumed medical decision-making authority and proceeded with PEG tube placement. In this case, the patient's son did not feel confident that he could make the "right" medical decision and he actively deferred decision making to his aunt, the patient's sister. Although the decision to give the patient a PEG tube was ultimately made by the surrogate, there seemed to be discomfort among members of the clinical care team regarding the patient's lack of assent. In both cases, the surrogates felt pressure to make a decision regarding PEG tube placement, which may have affected decision making.

Although both patient's outcomes could be considered ethically and medically acceptable; in both of these cases several challenges got in the way of decision making, including different prognoses offered by different medical providers, mistrust of the medical community, and multiple surrogates with differing opinions. Additionally, the patients' inability to assert their preferences and values about PEG tube placement posed additional challenges surrounding questions of substituted judgment and determining what was best for the patient. On the other hand, there were several components in these cases which helped facilitate decision making, including: identifying the most appropriate surrogate to make medical decisions, physicians who engaged in shared medical decision making with the surrogates, and including palliative care consultants to facilitate goals of care discussions.

Both of these cases highlight the importance of the role of the surrogate medical decision maker and high-quality goals of care conversations. In the case of Patient A, it is important to note the discrepancy of opinions between the clinical care teams on whether the patient should pursue a PEG tube and how these differing opinions complicated the decision making process for the patient's surrogate. Additionally, in both cases, some physicians and surrogates believed that death was the worst case outcome, while at the same time, some physicians and surrogates believed further decompensation and suffering would be the worst outcome. The concern that severe disability after stroke may be a fate worse than death is a highly prevalent belief and highlights the challenges that differing opinions on worse outcome present $(18,19)$. In both cases, the physicians worked with the patient's surrogates to make the decision regarding the PEG tube placement, despite their own personal feelings on prognosis and worst case outcome.

\section{Conclusions}

These cases demonstrate how physicians and surrogate 
medical decision makers approach the complex decision of PEG tube placement after stroke. Additionally, these cases highlight the complexities that can arise when multiple surrogates are involved in medical decision making and when multiple physician teams do not work together to present a more consistent message about the range and the most likely patient outcomes. Lastly, these cases show the vital importance of high-quality goals of care conversations about prognosis and quality of life when making complex medical decisions around artificial nutrition after severe stroke. High quality goals of care conversations include discussing patient preferences and values, as well as goals of care and treatment options that match the goals of medical care.

\section{Acknowledgments}

Funding: National Palliative Care Research Center, Kornfeld Scholars Grant Program.

\section{Footnote}

Reporting Checklist: The authors have completed the CARE reporting checklist. Available at http://dx.doi.org/10.21037/ apm-20-2094

Peer Review File: Available at http://dx.doi.org/10.21037/apm20-2094

Conflicts of Interest: All authors have completed the ICMJE uniform disclosure form (available at: http://dx.doi. org/10.21037/apm-20-2094). Dr. ARC received grant funding from the National Palliative Care Research Center as a Kornfield Scholar. Dr. ARC is the chair of the Ethics Committee and a member of the Public Policy Committee for the American Academy of Hospice and Palliative Medicine. Dr. AMT is supported by a Midcareer Investigator Award in Patient-Oriented Research (K24AG053794). The other authors have no conflicts of interest to declare.

Ethical Statement: The authors are accountable for all aspects of the work in ensuring that questions related to the accuracy or integrity of any part of the work are appropriately investigated and resolved. All procedures performed in studies involving human participants were in accordance with the ethical standards of the institutional and/or national research committee(s) and with the Helsinki Declaration (as revised in 2013). Written informed consent was obtained from the patient or their surrogate.
Open Access Statement: This is an Open Access article distributed in accordance with the Creative Commons Attribution-NonCommercial-NoDerivs 4.0 International License (CC BY-NC-ND 4.0), which permits the noncommercial replication and distribution of the article with the strict proviso that no changes or edits are made and the original work is properly cited (including links to both the formal publication through the relevant DOI and the license). See: https://creativecommons.org/licenses/by-nc-nd/4.0/.

\section{References}

1. Creutzfeldt CJ, Holloway RG. Treatment Decisions After Severe Stroke. Stroke 2012;43:3405-8.

2. Zier LS, Sottile PD, Hong SY, et al. Surrogate decision makers' interpretation of prognostic information: a mixedmethods study. Ann Intern Med 2012;156:360-6.

3. Holloway RG, Arnold RM, Creutzfeldt CJ, et al. Palliative and End-of-Life Care in Stroke: A Statement for Healthcare Professionals from the American Heart Association/ American Stroke Association. Stroke 2014;45:1887-916.

4. Creutzfeldt CJ, Robinson MT, Holloway RG. Neurologists as primary palliative care providers: Communication and practice approaches. Neurol Clin Pract 2016;6:40-8.

5. Crozier S, Santoli F, Outin H, et al. Severe stroke: prognosis, intensive care admission and withhold and withdrawal treatment decisions. Rev Neurol 2011;167:468-73.

6. Ojo O, Brooke J. The Use of Enteral Nutrition in the Management of Stroke. Nutrients 2016;8:827.

7. Dennis MS, Lewis SC, Warlow C. Effect of timing and method of enteral tube feeding for dysphasic stroke patients: A multicenter randomized controlled trial. Lancet 2005;365:764-72.

8. Ojo O. Balloon gastrostomy tubes for long-term feeding in the community. Br J Nurs 2011;20:34-8.

9. Sprigg N, Selby J, Fox L, et al. Very low quality of life after acute stroke: data from the efficacy of nitric oxide in stroke trial. Stroke 2013;44:3458-62.

10. Benejam B, Sahuquillo J, Poca MA, et al. Quality of life and neurobehavioral changes in survivors of malignant middle cerebral artery infarction. J Neurol 2009;256:1126-33.

11. Weil AG, Rahme R, Moumdjian R, et al. Quality of life following hemicraniectomy for malignant MCA territory infarction. Can J Neurol Sci 2011;38:434-8.

12. Albrecht GL, Devlieger PJ. The disability paradox: 
high quality of life against all odds. Soc Sci Med 1999;48:977-88.

13. Wendler D, Rid A. Systematic Review: The Effect on Surrogates of Making Treatment Decisions for Others. Ann Intern Med 2011;154:336-46.

14. Baile WF, Glober GA, Lenzi R, et al. Discussing disease progression and end of life decisions. Oncology (Williston Park) 1999;13:1021-31; discussion 1031-6, 1038.

15. Finestone HM, Blackmer J. Refusal to eat, capacity, and ethics in stroke patients: a report of 3 cases. Arch Phys Med Rehabil 2007;88:1474-7.

16. Holloway RG, Benesch CG, Burgin WS, et al. Prognosis and decision making in severe stroke. JAMA

Cite this article as: Comer AR, Williams LS, Bartlett SL, D'Cruz LE, Torke AM. Medical decision making about longterm artificial nutrition after severe stroke: a case report. Ann Palliat Med 2021;10(7):8484-8489. doi: 10.21037/apm-20-2094
2005;294:725-33.

17. Dugdale LS, Ridenour AA. Making sense of the Roman Catholic directive to extend life indefinitely. Hastings Cent Rep 2011;41:28-9.

18. Samsa GP, Matchar DB, Goldstien L, et. al. Utilities for major stroke: results from a survey of preferences among persons at increased risk for stroke. Am Heart J 1998;136:703-13.

19. Klein A, Kuehner C, Schwarz S. Attitudes in the general population towards hemi-craniectomy for middle cerebral artery (MCA) infarction. A population-based survey. Neurocrit Care 2012;16:456-61. 